\title{
An Enhancement of AODV with Multi-Radio in Hybrid Wireless Mesh Network
}

\author{
Mohammad Meftah Alrayes, Sanjay Kumar Biswash, Neeraj Tyagi, Rajeev Tripathi, \\ Arun Kumar Misra, and Sanjeev Jain
}

Department of Computer Science and Engineering, Motilal Nehru National Institute of Technology, Allahabad 211004, India

Correspondence should be addressed to Mohammad Meftah Alrayes; moha872@yahoo.co.uk

Received 29 April 2013; Accepted 11 July 2013

Academic Editors: M. Hopkinson and A. L. P. Rotondaro

Copyright (C) 2013 Mohammad Meftah Alrayes et al. This is an open access article distributed under the Creative Commons Attribution License, which permits unrestricted use, distribution, and reproduction in any medium, provided the original work is properly cited.

\begin{abstract}
AODV-MR (on-demand routing protocol with multi-radio extension) has been designed to support multi-radio interfaces; it uses more than one interface of the same mesh router or gateways for broadcasting duplicated control packets (i.e., RREQ, RRER, and HELLO message) or to rebroadcast it. We have modified AODV-MR, by allocating one interface in a dynamic manner for sending routing/control packets or data packets. This allocation of interfaces is based on type of mesh routers and traffic direction. The efficiency and effectiveness of the modification work have been evaluated compared with AODV-MR in terms of packet delivery ratio, routing packet overhead, end to end delay, and throughput.
\end{abstract}

\section{Introduction}

The wireless mesh networks are considered as a new attractive communication paradigm to provide IP connectivity, extending high-speed IP connectivity to the "last mile." It is still an ongoing problem of research area. Wireless mesh networks have several unique features like low cost, ease for rapid deployment, self-configuration, and self-healing. These characteristics encourage the use of wireless mesh network effectively. Wireless mesh networks can provide better and guaranteed QoS requirements in wide platform of application scenarios, such as disaster recovery, wireless broadband internet access, and intelligent transportation systems, and also in consumer demands for real-time services like video and audio services. Though several technologies have evolved for next generation communication, wireless mesh network has been gaining a significant attention of the researchers. Wireless mesh network can be extended for the connectivity, while the other access technology is unable to do this. The deployment of wireless mesh network is easy and cheap. There are three methods to classify the architecture of wireless mesh network: infrastructural backbone wireless mesh network, client wireless mesh network, and hybrid wireless mesh network [1] The proposed work focuses on hybrid mesh network, and our modification work considers architecture as shown in Figure 1, such that gateway routers are used for accessing the Internet or external network. The mesh routers have been divided into two parts, that is, backbone mesh routers and border mesh routers (BMR). The backbone mesh routers are representing the backbone of network. It is maintaining connectivity between mesh gateway and border mesh routers. It does not have a direct communication with mesh clients. Border Mesh Routers are used to forward traffic between backbone mesh routers and mesh clients.

The hybrid mesh network consists of two kinds of the traffic.

\subsection{Gateway Traffic}

(1) From multiple sources to single destination.

(2) From single source to multiple destinations.

The sources or destinations are either gateway or mobile mesh clients. 


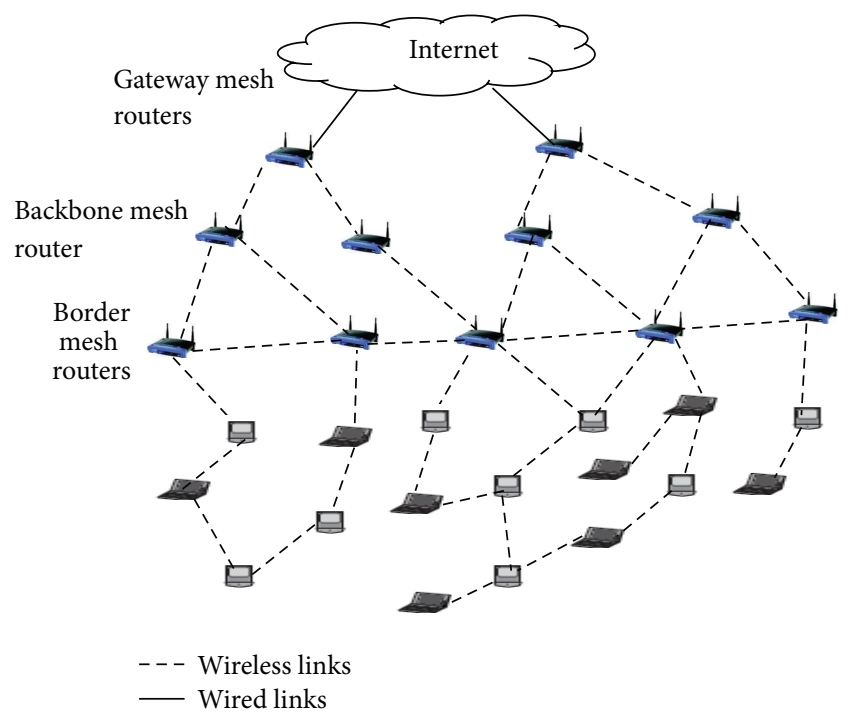

FIGURE 1: Architecture for hybrid wireless mesh network.

1.2. The Local Traffic or Peer to Peer Traffic Is Concerned with Traffic between Mobile Mesh Clients. It can be thus inferred that wireless mesh network borrows many characteristics from traditional Ad hoc network. Several research works has been carried out to apply the routing protocols of Ad hoc network to wireless mesh network [2-4], although these kinds of routing protocols required significant modification to enhance the effectiveness and efficiency to enhance the quality of service. The Ad hoc on-demand routing protocol (AODV), and its extension multi-radio extension (AODVMR) [2], has been used for hybrid wireless mesh network. However these kinds of routing protocol have highly routing overhead by broadcasting routing control packets to more than one interface. Thus it causes unnecessary processing for routing packets, bandwidth utilization, which in turn degrades the overall network performance. The AODV-MR assigns an interface (channel) in a random manner for sending data packets, which is not useful, because of unpredictable changes in wireless channel environment.

QoS of any network can be enhanced using cross-layer design. The cross layer has two types: loosely coupled and tightly coupled. In loosely coupled, the parameters in one protocol layer are forwarded to another protocol layer; for example, interference level in physical layer can report to the network layer or MAC layer. In tightly coupled cross layer, all layers merge together in single layer; for example, MAC routing and physical protocols can be optimized in single layer [5]. In this paper loosely coupled scheme has been used, such that MAC layer passes channel status to network layer and the network layer decides the channel to be used.

The modification work eliminates routing overheads by selecting a proper single interface in a dynamic manner, instead of using all interfaces. The other interfaces are then used only for sending data packets, which in turn enhances the efficiency of the network.

The choice of interface (channel) to communicate between two successive nodes will not affect the routing metric.
In the proposed work, the interflow and intraflow interference will reduce to achieve a good performance in the overall network. Further, the proposed work neither addressed channel assignment problem nor modified any routing packet header.

The remainder of the paper is organized as follows: Section 2 discusses the related work. The motivation for proposed work has been discussed in Section 3. Section 4 presents proposed work. Proposed modification discusses in details in Section 5, Model formulation presents in Section 6, The simulation environment for the proposed work has been discussed in Section 7. The detailed analysis for results and discussions are given in Section 8 , followed by conclusion and references.

\section{Related Work}

Routing protocols are key challenges in hybrid wireless mesh network. A number of routing protocols have been introduced to date. Routing in mesh networks is one of the key challenges and is generally achieved using Ad hoc routing protocols. There are two well-known Ad hoc routing protocols: (1) proactive (table-driven) like DSDV [6] and (2) reactive (on-demand) routing protocols like DSR [7] and AODV [8]. These protocols are developed to work in hybrid wireless mesh networks. Most of research works proposed for hybrid wireless mesh network is based on reactive and proactive routing protocols only.

In [3], the author has modified DSDV and DSR with new routing metric named ETX, which is helpful in finding paths with high throughput in multi hop wireless networks. The author of [4] proposed a routing protocol derived from reactive routing protocol (i.e., DSR). It has been combined with a new metric named weighted cumulative expected transmission time (WCETT). It has been assumed that all nodes are static, and each node is equipped with one or more 802.11 radios with different noninterfering channels for a long time. The limitation of this proposal is that it does not take interflow interference into consideration, which might result in a poor throughput. In [9] the authors presented a routing scheme called AODV-ST, which is a modification of AODV, and they focused on traffic that flows from routers to gateway. It is based on infrastructure wireless mesh network and employs a proactive approach to discover routes between routers and gateway. It splits multi-radio interfaces into two sets. First, the subset of radio interface is used to communicate with users and the other subset to relay traffic towards gateway. The author of [10] has proposed a multichannel routing protocol for wireless mesh network for dynamic wireless mesh network. The proposed [10] methodology is a changed version of on-demand routing protocol. Their proposed work classified interfaces into two parts: one is fixed interface and the other is switchable interface. The fixed interface and channel have been assigned to receive a packet from neighbors, while a switch interface is used for sending packets to neighbors. Whenever a node desires to send packets to a particular neighbor, it will check the table to find a channel that is used as a fixed channel by neighbor. 
The switchable interface will switch to the same channel that is used as a fixed channel of the received node (i.e., next hop). The authors have also suggested using HELLO message to exchange fixed channel information between neighbors. This proposal might not be efficient in case of high traffic load where the switching interface will be high. In [11], the authors proposed a new metric based on interference to find the routing path. It reduces interflow and intraflow interferences and incorporated this metric to support multi-radio networks for AODV routing protocol. The authors in [12] focused on IEEE 802.11-based wireless mesh networks with stationary nodes and proposed a metric called bottleneck link capacity (BLC), which accounts for the link quality, the interference among links, and the traffic load on the links: the proposed metric has behavior similar to that of on-demand routing protocol. In [13], the authors proposed extensions to the wellknown ad hoc on-demand distance vector (AODV) routing protocol with the aim to discover and exploit multiple links in wireless mesh networks. In [14], the authors suggested to use queue lengths as metric and incorporated it with AODV routing protocol. In [15], the authors proposed an on-demand channel reservation scheme to reserve some of mesh router radio interfaces (i.e., channels) to support the gateway traffic while the remaining interfaces can be used to support the local traffic.

\section{Motivations}

The multi-radio AODV is extension of AODV protocol, and it has been proposed for hybrid wireless mesh network. During the route discovery of mesh node (i.e., gateway, mesh routers, and mesh clients), the mesh client uses single radio interface. It has been used for broadcasting RREQ packets, while gateway and mesh routers will use all interfaces to broadcast the same RREQ packets; if it is a source node, while mesh routers are receiving duplicated RREQ from all interfaces, it will check for the duplicity (it is a fresh packet or received previously). For the fresh packet, it will rebroadcast it again in all interfaces except the interface from which it received RREQ packets; otherwise it will drop it [8]. The mechanism of broadcasting a duplicated packet of RREQ to more than one interface will cause an increase in a number of RREQ packets in the overall networks. The number of RREQ will be very high in the case of source node not finding the route to the destination. Consequently, the load on channels attached with interfaces will increase due to these packets. It means that the total routing overhead is increased; end to end delay will increase, due to waiting time in queue. Figure 2 shows an example of receiving multiple control packets such that node A is source node; B and D are intermediate nodes. Node $D$ receives the same RREQ packets from $A$ and $B$, and node $D$ receives 3 duplicates of RREQ packets from node A and 2 duplicates of RREQ packets from B, so that total duplicates of RREQ will be 5 packets.

For maintaining route connectivity, mesh nodes use periodic HELLO message. The gateway and intermediate mesh routers will broadcast this duplicate message through all interfaces in every one hope distance in the path; this mechanism helps to increase the total number of routing

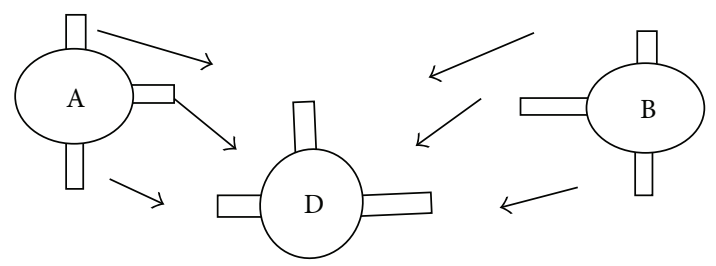

FIGURE 2: Router D receives the same duplicated broadcasting packets from $\mathrm{B}$, and $\mathrm{A}$.

overheads, and it increases the length of queue at each interface. In the case of route failure/alternate route failure, the RERR packets are resent by all interfaces in mesh routers that are involved in the path with increasing the number of route failures and the number of route requests, and error packets will be increased. Thus route discovery process is increased, and it will degrade the performance of the network. Duplicated control packets through more than one interface will result in unnecessary processing mechanism for control packets at routing layer. Whenever a mesh node receives control packets it will check whether it has received this packet before or not; the checking process is repeated for every received control packet, and even it is duplicated. This process of duplicated packets will lead to increase the waiting time for other packets; moreover if the waiting time of the packets is more than certain period of time like $30 \mathrm{~s}$, these packets will drop to prevent buffering of packets indefinitely [16]. The queue interface is used to buffer the data packets and control packets for transmission and retransmission. The increasing number of control packets will degrade in utilization of total queue interfaces. When high numbers of control packets are arriving at queue interface, the queue buffer size is reduced. At a particular time the queue buffer might be full, and the possibility to drop next arrived packets in queue is very high due to buffer overflow. When data packets have been dropped, the ACK packet will not be transmitted from receiver data packets to the sender. The routing layer will receive notification by MAC layer for route break. This causes incensement in contention period for acquiring the channel. All these said issues will be reflected in terms of QoS, like increasing end to end delay, increasing overhead, and reducing throughput in all over network. With this motivation we are proposing our technique for AODV to enhance the better QoS.

\section{Proposed Work}

The modification work considers a network model, which is an enhancement of AODV-MR routing protocol, and details the working methodology of the proposed technique.

4.1. Model Description. In the proposed work following assumptions have been made.

(i) All gateways and mesh routers have identical number of multi-radio interface, and channel allocation.

(ii) Each radio interface has its own MAC and physical layers. These radio interfaces are considered to be nonoverlapping channels. 
(iii) There are no two interfaces sharing in a common channel. Hence, these radio interfaces are independent in communications as shown in Figure 3.

(iv) Mesh clients have a single interface tuning statically to a single non-overlapping channel as shown in Figure 4.

In case of IEEE $802.11 / \mathrm{b} / \mathrm{g}$ there are three non overlapping channels while in case of 802.11/a there are twelve nonoverlapping channels [17-19]; actually each radio interface might be tuned to multiple channels. In this paper, channel assignment problem in wireless mesh network has not been addressed. We consider it for simplicity in design and application. Our proposed work based on interactions between MAC layer and routing layer in cross-layer scheme. We are considering this architecture for the following benefits:

(1) simple and provides optimum full network connectivity;

(2) eliminates channel switching overhead, where larger switching time will degrade the network performance;

(3) prevents latency and congestion, due to highly channel negations;

(4) synchronization with other nodes for the selection of channel is not required;

(5) no need to modify MAC layer protocol;

(6) it is possible to implement our architecture using IEEE 802.11 hardware;

(7) keeps continuously measuring all channel states and buffer Queue interface length;

(8) it can be getting instant information about the load and interference level for each channel in mesh routers and gateways;

(9) no need for any kind of virtual MAC protocol to coordinate communication in all channels as proposed in [17];

(10) mesh routers can send and receive simultaneously so that it can form a full duplex exchange;

(11) the rendezvous problem (i.e., pair of nodes are not able tuned to common channel for exchange packets) and the multichannel hidden terminal problem are not present;

(12) prior knowledge about network topology is not necessary for the selection of interface (i.e., channel);

(13) no need to modify control packets of standard AODV.

\section{Proposed Modification}

We have proposed a mechanism that modified multi-radio AODV in hybrid wireless mesh network (M-AODV-MR). It takes traffic exchange between the mesh router, gateway and mesh client, and between mesh clients themselves into consideration. Our proposed work will not modify any AODV control packets. So there is no further overhead due to

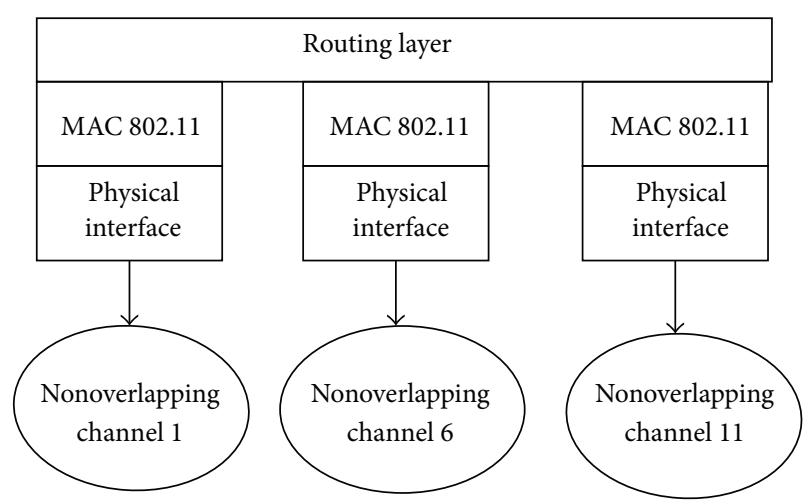

FIGURE 3: Layout of our proposed work of mesh router.

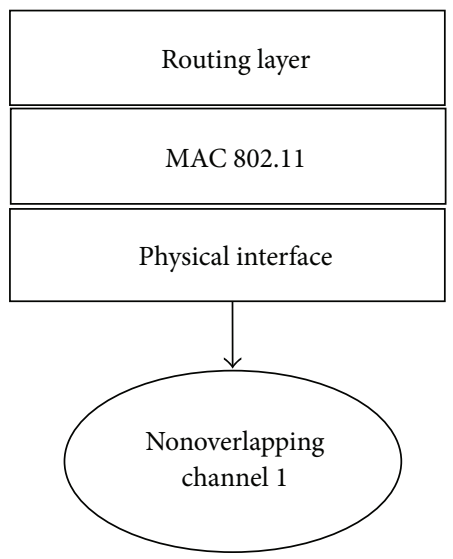

FIGURE 4: Layout of mesh client.

the increase in the size of the packets. The proposed methodology integrates channel assignment with AODV routing algorithm. The MAC layer gathers information for each channel and passes it to routing layer in cross-layer manner. Based on it, routing layer will choose an appropriate channel to forward data packets or control packets to next hope. In MAODV-MR, the traffic is classified into three directions for the case of route discovery, during exchange data packet, hello message, route reply, and route failure. Source address and destination address are used to distinguish between traffic directions in the network. Figures 5 and 6 illustrate the mechanism of sending packet, and they are classified into the following three ways:

(1) FGTMC (from gateway to mesh client);

(2) FMCTG (from mesh client to gateway);

(3) FMCTMC (from mesh client to mesh client).

5.1. From Gateway to Mesh Client. When mesh gateway intends to send data to mesh client, it does not have a fresh route. To find out the route it will broadcast RREQ packets. Before that, the routing layer will collect the information about the channel utilization (i.e., it is a fraction of channel time in which channel sensing is busy) from each individual using MAC layer. The contention in shared channel is 


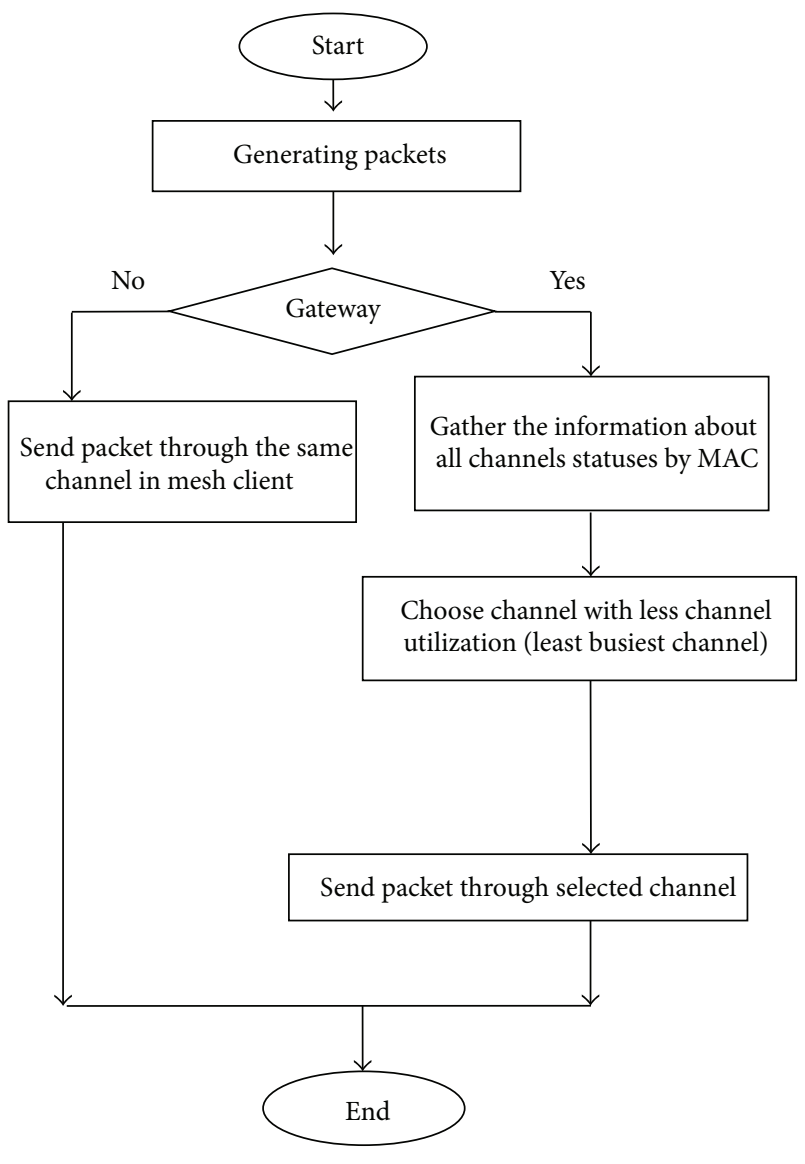

FIGURE 5: Flowchart for sent packet.

common nature of wireless networks. These contentions of channel give indication of intraflow and interflow interference. The increase in the flow traffic at neighboring nodes will increase the contention channel as the number of packets at queue will be more and the waiting time at queue interface will increase. Based on channel utilization information, the gateway forwards the RREQ packets into less channel utilization (i.e., less busiest channel). Because mesh gateway and mesh router have the same number of interfaces, those interfaces allocate the same channels; the control packets can be exchanged as single common channel.

When backbone mesh router receives RREQ as intermediate node in route, it will check destination address in RREQ packet. In case destination address is gateway (i.e., node from external network) or mesh client, then it will apply the same role as gateway routers to choose the interface (channel). When the border mesh router and mesh client receive RREQ packets as intermediate node, it will rebroadcast RREQ via the same channel of mesh clients.

5.2. From Mesh Client to Gateway. When mesh client sends data to gateway and it has no route to the gateway, then it will broadcast RREQ via its default channel. All mesh clients that receive RREQ packets will rebroadcast it on default channel. Once the border mesh router or backbone mesh router receives RREQ, it will check the destination address to

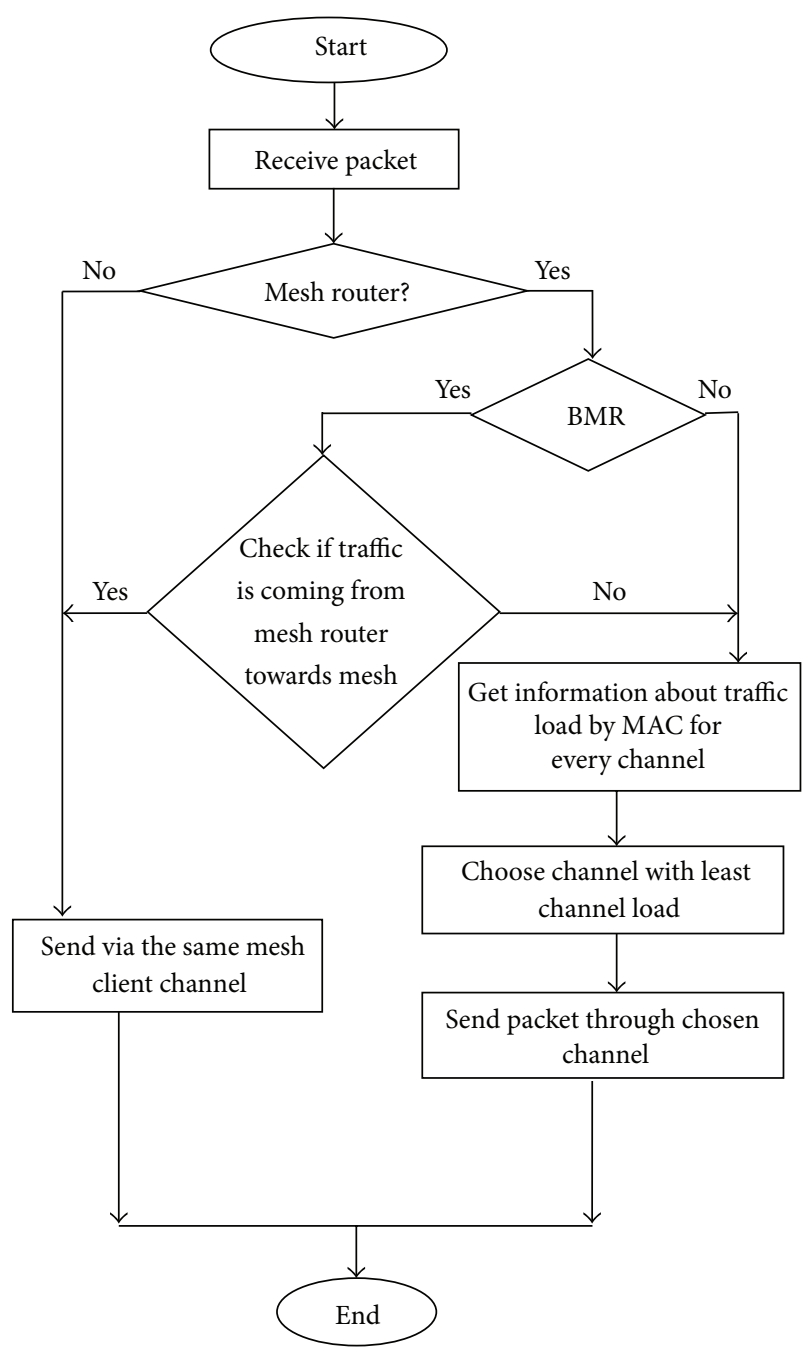

FIGURE 6: Flowchart for the received packet.

ensure that the destination address is gateway, and then it will rebroadcast RREQ packet via the interface that is attached with less channel load (less busiest channel).

5.3. From Mesh Client to Mesh Client. In case of not having a route and mesh client intending to send data to the same mesh client, it will broadcast RREQ via its common channel as AODV does in the traditional manner. At some time, due to the nature of broadcasting, a backbone mesh router might receive RREQ packet, where its destination address and source address are mesh client addresses. Thus, it is not necessary to rebroadcasting this RREQ packet among mesh routers and gateway as this RREQ packet might create storm or congestion at particular path. In this case backbone mesh router will suppress the RREQ from being rebroadcast between backbone mesh routers. It helps to reduce overhead in overall network and reduce bandwidth utilization that occurs during a period of routing control packets. The reduction of bandwidth might be utilized for sending data packets. In our modification technique the route reply will be same as the route discovery, that is, the destination 


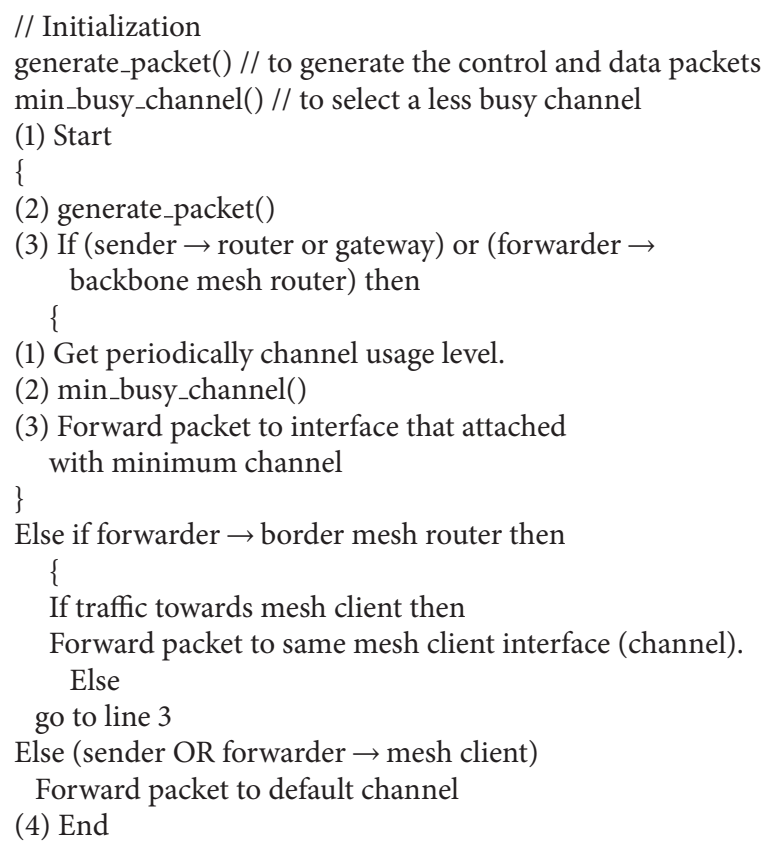

(1) Get periodically channel usage level.

(2) min_busy_channel()

(3) Forward packet to interface that attached with minimum channel

Pseudocode 1: Pseudocode for the proposed technique.

node will unicast RREP packet through a suitable interface as mentioned in route discovery process. During the maintains route connectivity, backbone mesh router and gateway will use the same procedure as route discovery for sending a periodic HELLO message, while border mesh router will the use same channel as in mesh client. In the route failure, the border mesh router will be checked in the following manner. If the source of a route is gateway, then it will send to precursor mesh routers via best interface (less channel utilization); otherwise it will use the same channel mesh client in case the source is mesh client. After the route has been established, data packets will be transmitted through the interface (i.e., channel) dynamically, from hop to hop, so that, possibility of using channel diversity will be high.

5.4. Pseudocode for the Proposed Technique. For more details see Pseudocode 1.

\section{Model Formulation}

In this section we have formulated our proposed model. For this purpose we have introduced some symbols, and they are available in Table 1 with description. In AODV routing protocol, there are three prime requirements, and they are route discovery $(D)$, route error (RE) and route maintenance (RM). The representations of them are " $D$," "RE," and " $M$," respectively. The Markov state diagram of these states can be represented as follows (as shown in Figure 7).

Based on it we can compute the possibility of different cases for the movement, and they can be represented as follows.
TABLE 1: Notation and its description.

\begin{tabular}{ll}
\hline Symbols & Description \\
\hline$\nu / \rho / L$ & $\begin{array}{l}\text { Velocity of the mobile node/density of } \\
\text { nodes/perimeter of the area }\end{array}$ \\
\hline & $\begin{array}{l}\text { Rate of state change from route- } \\
\text { discovery-to-route-discovery/route- } \\
\text { discovery-to-route-error/route-error- } \\
\text { to-route-error/route-discovery-to- } \\
\text { route-error }\end{array}$ \\
\hline $\mathrm{RC}_{D}^{D} / \mathrm{RC}_{D}^{\mathrm{RE}} / \mathrm{RC}_{\mathrm{RE}}^{\mathrm{RE}} / \mathrm{RC}_{D}^{M}$ & $\begin{array}{l}\text { Total delay/delay at route discovery } \\
\text { state/delay at route error state/delay at } \\
\text { route maintenance state }\end{array}$ \\
\hline$\pi_{D} / \mathrm{D}_{D} / \mathrm{D}_{\mathrm{RE}} / \mathrm{D}_{M}$ & $\begin{array}{l}\text { Probability for the route discovery } \\
\text { phase/probability for the route } \\
\text { maintenance phase }\end{array}$ \\
\hline $\mathrm{SP}_{D}$ & $\begin{array}{l}\text { Size of data packets } \\
\mathrm{Bd}\end{array}$ \\
\hline Bandwidth of the wireless network \\
\hline $\mathrm{Hd}_{D}^{\mathrm{RE}} / \mathrm{Hd}_{\mathrm{RE}}^{M} / \mathrm{Hd}_{M}^{D}$ & $\begin{array}{l}\text { Hop distance from } \\
\text { route-discovery-to-route-error/route- } \\
\text { error-to-route-maintenance/route- } \\
\text { maintenance-to-route-discovery }\end{array}$ \\
\hline $\mathrm{P}_{\mathrm{RE}} / \mathrm{P}_{M} / \mathrm{P}_{D}$ & $\begin{array}{l}\text { Processing time at } \\
\text { route-error/maintenance/discovery }\end{array}$ \\
\hline $\mathrm{PR}_{D} / \mathrm{PR}_{\mathrm{RE}}$ & $\begin{array}{l}\text { Propagation time to } \\
\text { route-discovery/route-error }\end{array}$ \\
\hline &
\end{tabular}

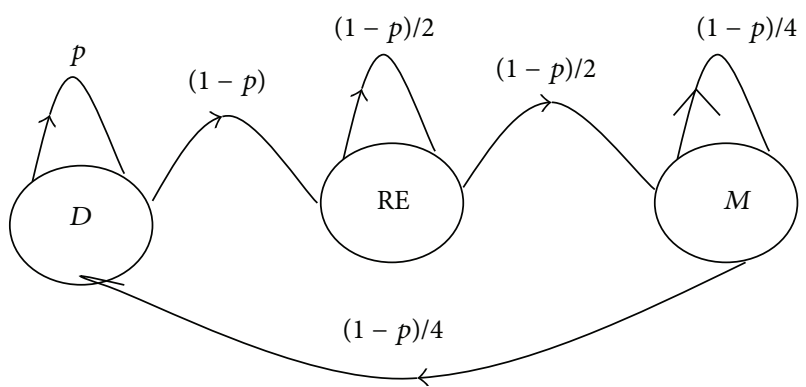

FIGURE 7: Markov state diagram for states.

Case 1. The object is changing the state from route discovery to route break.

Case 2. The object is changing the state form route error to route maintenance phase.

Case 3. The object is changing the state from route maintenance to route discovery.

Case 4. The object does not change its state at route discovery.

Case 5. The object does not change its state at route error.

Case 6. The object does not change its state at route maintenance.

With the above discussion we can analyze the changes in states, from another. The average number of rates of phase 
changes can be computed as $\Omega=(\nu \rho L) / \pi[20,21]$. The possibility of various kinds of state change can be computed as follows.

(1) The object does not change the phase $\left(\mathrm{RC}_{D}^{D}\right) /\left(\mathrm{RC}_{D}^{D}+\right.$ $\left.\mathrm{RC}_{D}^{\mathrm{RE}}\right)$.

(2) Its moves to route break $\left(\mathrm{RC}_{D}^{\mathrm{RE}}\right) /\left(\mathrm{RC}_{D}^{\mathrm{RE}}+\mathrm{RC}_{D}^{D}\right)$.

(3) Route break to maintenance can be calculated as $\left(\mathrm{RC}_{\mathrm{RE}}^{\mathrm{RE}}\left(\mathrm{RC}_{D}^{\mathrm{RE}}+\mathrm{RC}_{D}^{D}\right)\right) /\left(\mathrm{RC}_{D}^{\mathrm{RE}}\right)$.

(4) Route maintenance to route discovery can be computed as $\left(\mathrm{RC}_{D}^{\mathrm{RE}}-\mathrm{RC}_{\mathrm{RE}}^{\mathrm{RE}}\left(\mathrm{RC}_{D}^{\mathrm{RE}}+\mathrm{RC}_{D}^{D}\right)\right) /\left(\mathrm{RC}_{D}^{\mathrm{RE}}\right)$, where $\mathrm{RC}$ is the range from one phase to another.

In this paper, the effectiveness and efficiency have been checked by end to end delay, packet delivery fraction (PDF), packet overhead, and throughput. All of the above factors fully depend on hop-by-hop delay, latency, and average number of path/packet losses based on distance and hops. So here we will analyze the delay, latency, and average number of path/packet losses.

Delay and latency metrics depend on the route discovery delay, and it is the time required to deliver the first packet to the destination. In this section we compute the route discovery delay for the proposed system. The delay of the proposed system can be computed as [20]

$$
\mathrm{D}=\pi_{D} \cdot \mathrm{D}_{D}+\pi_{\mathrm{RE}} \cdot \mathrm{D}_{\mathrm{RE}}+\pi_{M} \cdot \mathrm{D}_{M}+S_{t}
$$

where $S_{t}$ is the time required to observe the less queue length at interfaces and the amount of the $S_{t}$ is a very low.

The latency (L) can be computed as follows in [20]

$$
\begin{aligned}
\mathrm{L}= & \pi_{D} \times\left(\mathrm{RC}_{D}^{D}+\mathrm{RC}_{D}^{\mathrm{RE}}\right)+\pi_{\mathrm{RE}} \times\left(\mathrm{RC}_{\mathrm{RE}}^{\mathrm{RE}}+\mathrm{RC}_{\mathrm{RE}}^{M}\right) \\
& +\pi_{\mathrm{RE}} \times\left(\mathrm{RC}_{M}^{M}+\mathrm{RC}_{M}^{D}\right),
\end{aligned}
$$

where

$$
\mathrm{D}_{D}=\left(\frac{\mathrm{SD}_{P}}{\mathrm{Bd}}+\mathrm{RC}_{D}^{D}\right) \times \mathrm{Hd}_{D}^{\mathrm{RE}}+\mathrm{P}_{D}+\mathrm{P}_{R} \times \mathrm{Hd}_{D}^{\mathrm{RE}} .
$$

Hop distance travelled, to change the phase

$$
\begin{gathered}
\mathrm{D}_{\mathrm{RE}}=\left(\frac{\mathrm{SD}_{P}}{\mathrm{Bd}}+\mathrm{RC}_{\mathrm{RE}}^{\mathrm{RE}}\right) \times \mathrm{Hd}_{\mathrm{RE}}^{M}+\mathrm{P}_{\mathrm{RE}}+\mathrm{P}_{\mathrm{R}} \times \mathrm{Hd}_{\mathrm{RE}}^{M}, \\
\mathrm{D}_{M}=\left(\frac{\mathrm{SD}_{P}}{\mathrm{Bd}}+\mathrm{RC}_{M}^{D}\right) \times \mathrm{Hd}_{M}^{D}+\mathrm{P}_{M}+\mathrm{P}_{R} \times \mathrm{Hd}_{D}^{\mathrm{RE}},
\end{gathered}
$$

where

$$
\begin{aligned}
\mathrm{RC}_{D}^{D}= & \frac{\mathrm{SR}_{\mathrm{RREQ}}+\mathrm{SR}_{\mathrm{RREP}}}{\mathrm{Bd}}+\pi_{D} \\
& +\left(\frac{\mathrm{SR}_{\mathrm{RREQ}}+\mathrm{SR}_{\mathrm{RREP}}}{\mathrm{Bd}}+\pi_{D}\right) \\
& \times\left(\mathrm{Hd}_{D}^{D}-1\right)+\mathrm{PR}_{D},
\end{aligned}
$$

$$
\begin{aligned}
\mathrm{RC}_{D}^{\mathrm{RE}}= & \frac{\mathrm{SR}_{\mathrm{RREQ}}+\mathrm{SR}_{\mathrm{RREP}}}{\mathrm{Bd}}+\mathrm{RC}_{D}^{D} \\
& +\left(\frac{\mathrm{SR}_{\mathrm{RREQ}}+\mathrm{SR}_{\mathrm{RREP}}}{\mathrm{Bd}}+\pi_{D}^{\mathrm{RE}}\right) \\
& \times\left(\mathrm{Hd}_{D}^{\mathrm{RE}}-1\right)+\mathrm{PR}_{\mathrm{RE}}, \\
\mathrm{RC}_{\mathrm{RE}}^{\mathrm{RE}}= & \frac{\mathrm{SR}_{\mathrm{RREQ}}+\mathrm{SR}_{\mathrm{RREP}}}{\mathrm{Bd}}+\mathrm{RC}_{D}^{\mathrm{RE}} \\
& +\left(\frac{\mathrm{SR}_{\mathrm{RREQ}}+\mathrm{SR}_{\mathrm{RREP}}}{\mathrm{Bd}}+\pi_{\mathrm{RE}}^{\mathrm{RE}}\right) \\
& \times\left(\mathrm{Hd}_{\mathrm{RE}}^{\mathrm{RE}}-1\right)+\mathrm{PR}_{\mathrm{RE}}, \\
\mathrm{RC}_{\mathrm{RE}}^{D}= & \frac{\mathrm{SR}_{\mathrm{RREQ}}+\mathrm{SR}_{\mathrm{RREP}}}{\mathrm{Bd}}+\mathrm{RC}_{\mathrm{RE}}^{D} \\
& +\left(\frac{\mathrm{SR}_{\mathrm{RREQ}}+\mathrm{SR}_{\mathrm{RREP}}}{\mathrm{Bd}}+\pi_{\mathrm{RE}}^{D}\right) \\
& \times\left(\mathrm{Hd}_{\mathrm{RE}}^{D}-1\right)+\mathrm{PR}_{\mathrm{RE}} \cdot
\end{aligned}
$$

The next important metric is queue waiting time; it should be calculated in the following manner.

The probability that the stationary process is in state $i$ (containing $i$ customers, including those in service) is $\pi_{i}=$ $(1-p) p^{i}$, where $p$ is the probability for packet arriving in the queue of interface. The busy period is the time period measured between the customers that arrives to an empty system until the instant a customer departs leaving behind an empty system. The busy period has Poisson probability density function, and it can be measured as follows. The $f(t)$ is a time function:

$$
f(t)= \begin{cases}\frac{1}{t \sqrt{p}} e^{-(\lambda+\mu)^{t}} I_{1}(2 t \sqrt{\mu \lambda}) & t \geq 0 \\ p & \text { otherwise }\end{cases}
$$

$$
f\left(t_{2}\right)= \begin{cases}(\lambda-\mu) e^{(\mu-\lambda) t} & \\ 0 & \text { otherwise. }\end{cases}
$$

The final queue waiting time can be computed as

$$
f(T)= \begin{cases}\frac{1}{t \sqrt{p}} e^{-(\lambda+\mu)^{t}} I_{1}(2 t \sqrt{\mu \lambda}) & \\ +(\lambda-\mu) e^{(\mu-\lambda) t} & t \geq 0 \\ p & \text { otherwise. }\end{cases}
$$

Our proposed technique is an optimal one so $f(T)$ can be calculated as

$$
f(T)=\operatorname{Min} \begin{cases}\frac{1}{t \sqrt{p}} e^{-(\lambda+\mu)^{t}} I_{1}(2 t \sqrt{\mu \lambda}) & \\ +(\lambda-\mu) e^{(\mu-\lambda) t} & t \geq 0 \\ p & \text { otherwise, }\end{cases}
$$

where $\lambda$ and $\mu$ have the normal meaning for queue system. 
Assume that node $n_{i}$ is available for routing with probability $p\left(n_{i}\right)$ and the link $\left(n_{i}, n_{i+1}\right)$ is available with probability $p_{l}\left(n_{i}, n_{i+1}\right)$ and path availability $p_{p}\left(p_{l}, p_{d}\right)$ from node $n_{l}$ to node $n_{d}$, and then the path availability can be calculated as

$$
p_{p}\left(n_{i}, n_{d}\right)=\prod_{1=1}^{d-1} p_{(n+1)} \times p_{\left(n_{i}, n_{(n+1)}\right)}
$$

$p$ represents the error ratio of the wireless networks. The derivation of $p$ can be represented as follows. The error $p$ of $\varepsilon$ is the probability of erroneous classification; namely, $\varepsilon=p(\psi(X) \neq Y)$. This true error is relative to a featurelabel distribution $f_{X, Y}$, and it equals the expected absolute difference between the label and classification, $E[|Y-\psi(X)|]$.

It can also be decomposed as

$$
\varepsilon=c \varepsilon^{0}+(1-c) \varepsilon^{1}
$$

where

$$
\varepsilon^{0}=p(\psi(X)=1 \mid Y=0)=\int_{\psi(X)=1} f_{X \mid Y}(X \mid 0) d x .
$$

Our proposed techniques follow the minimum of the queue length. So

$$
\text { Optimized Function } F(\text { Pro })=\lim _{X \rightarrow \operatorname{Min}} \varepsilon^{0}
$$

\section{Simulation Environment}

The effectiveness and efficiency of the proposed work have been compared with AODV-MR [2], using the NS-2 simulator version 2.33 [22]. This simulation environment has been configured and modified with multi-radio interfaces to simulate our proposed work and AODV-MR; we build up a simulation topology like an architecture of hybrid wireless mesh network in Figure 1. Here we are assuming the following attributes.

(i) Numbers of static mesh nodes have been fixed in all simulation scenarios.

(ii) Numbers of mobile mesh clients have been considered as variant when studying the effect of network size between 5 mesh client nodes to 65 mesh clients.

(iii) Pause time for mobile mesh client in all scenarios is zero.

(iv) Flat topology has been used in all simulation scenarios.

(v) Every radio interface works on half-duplex mode.

(vi) The traffic flows are considered from gateway to the mobile mesh clients, from mobile mesh clients to gateway, and between mesh clients themselves.

\begin{tabular}{|c|c|}
\hline Parameter & Value \\
\hline Application type & Constant bit rate (CBR) \\
\hline Transport type & $\begin{array}{l}\text { User datagram protocol } \\
\text { (UDP) }\end{array}$ \\
\hline Routing protocols & $\begin{array}{l}\text { AODV-MR and proposed } \\
\text { (M-AODV-MR) }\end{array}$ \\
\hline Simulation time & 250 seconds \\
\hline Packet size & 512 bytes \\
\hline Simulation area & $1200 \mathrm{~m} \times 1200 \mathrm{~m}$ \\
\hline $\begin{array}{l}\text { Number of static mesh } \\
\text { routers (i.e., assumed) }\end{array}$ & 18 \\
\hline Mobility model & Random way point \\
\hline Propagation model & Two ray ground \\
\hline MAC layer & $\begin{array}{l}\text { IEEE 802.11DCF with } \\
\text { RTS/CTS }\end{array}$ \\
\hline Antenna model & Omnidirectional antenna \\
\hline $\begin{array}{l}\text { Number of radio interfaces } \\
\text { in each mesh router }\end{array}$ & 3 \\
\hline $\begin{array}{l}\text { Number of channels in each } \\
\text { mesh router }\end{array}$ & $\begin{array}{l}3 \text { channel numbers and } \\
\text { types ( } 1,6 \text {, and } 11802.11 b \\
\text { channels) }\end{array}$ \\
\hline $\begin{array}{l}\text { Number of radio interfaces } \\
\text { in each mesh client }\end{array}$ & $\begin{array}{l}1 \text { channel type }(802.11 b \\
\text { channel) }\end{array}$ \\
\hline $\begin{array}{l}\text { Number of channels in each } \\
\text { mesh client }\end{array}$ & 1 channel number (1) \\
\hline Transmission range & $250 \mathrm{~m}$ \\
\hline Carrier sense & $550 \mathrm{~m}$ \\
\hline $\begin{array}{l}\text { Maximum speed mobility of } \\
\text { mesh clients }\end{array}$ & $10 \mathrm{~m} / \mathrm{s}$ \\
\hline Pause time & 0 \\
\hline
\end{tabular}

7.1. Simulation Parameters. Table 2 shows all parameters for the simulation environment.
TABLE 2: Simulation parameters.

7.2. Performance Metrics. To analyze the performance of the proposed approach, we have performed several experiments with the following metrics.

For each scenario, we have processed between 50 and 75 independent simulations with different mobility scenarios of mesh clients. For the compatibility we are calculating the average of these results.

7.2.1. Packet Delivery Fraction (PDF). It calculates the ratio between the total amount of successfully received data packet and the total number of generated data packets. This metric is presenting the span of reliability and efficiency of each routing protocol, and it can be computed as

$$
\mathrm{PDF}=\frac{\sum_{i=1}^{n} P_{r}[i]}{\sum_{j=1}^{n} P_{t}[j]} \times 100
$$

where $n$ is the total number of nodes in network.

$P_{r}[i]$ is the total number of data packets, successfully received by node $i$ during simulation experiment. $P_{t}[j]$ is the total number of data packets that has been generated and sent by node $j$ during simulation experiment. 
7.2.2. Average Route Overhead. It indicates the ratio between total amounts of generating routing control packets of each successful received data packet to the total number of successfully received data packets:

$$
\text { Average Routing Overhead }=\frac{\sum_{i=1}^{n} P_{\text {overhead }}[i]}{\sum_{i=1}^{n} P_{r}[j]},
$$

where $P_{\text {overhead }}[i]$ is the total number of routing control packets generated for data packet by node $i . P_{r}[j]$ is the total number of successfully received data packets by node $j$.

7.2.3. Average Throughput. The average number of data packets delivered during a session is

$$
\text { Average Throughput }=\frac{\sum_{i=1}^{n} P_{r}[i]}{T_{r}-T_{s}},
$$

where $T_{r}$ is the time at which packet is received by receiver. $T_{s}$ is the time at which packet is sent by sender.

7.2.4. Average End to End Delay (AEED). The total time taken by all data packets from source to destination over total number of successful received data packets $(N)$ is

$$
\text { AEED }=\text { End_to_End_Delay } \times 1000(\mathrm{~ms}),
$$

where

$$
\begin{gathered}
\text { End_to_End_Delay }=\frac{\text { TDT }}{\sum_{i=1}^{N} P_{r}[i]}, \\
\text { TDT }=\mathrm{TDT}+\sum_{i=0}^{N} \operatorname{delay}[i], \\
\text { delay }[i]=T_{r}[i]-T_{s}[i] .
\end{gathered}
$$

\section{Results and Discussion}

The simulation results are obtained under several experiments with different mobilities of mesh clients. The results for proposed work have been compared with the results of AODV-MR protocol [8]. For the experiments we are dealing with end to end delay, packet delivery ratio, and throughput and normalization route overhead.

8.1. Scenario 1: Varying Number of Mesh Clients. In this scenario, we have varied the network size, by varying the number of mobile mesh clients from 5 to 65 . The number of the flow connections between sources and designations is fixed to 20, and transmission rate is 20 packets/second. The results of this scenario are shown in Figures 8, 9, 10, and 11.

In Figure 8, we can identify that with an increase in the number of mobile mesh clients end to end delay will also increase continuously (i.e., direct relationship), because the number of hops between gateway to mesh clients and mesh clients themselves will be high.

Moreover AODV-MR uses all interfaces to send multiple copies of control packets in mesh routers. These packets

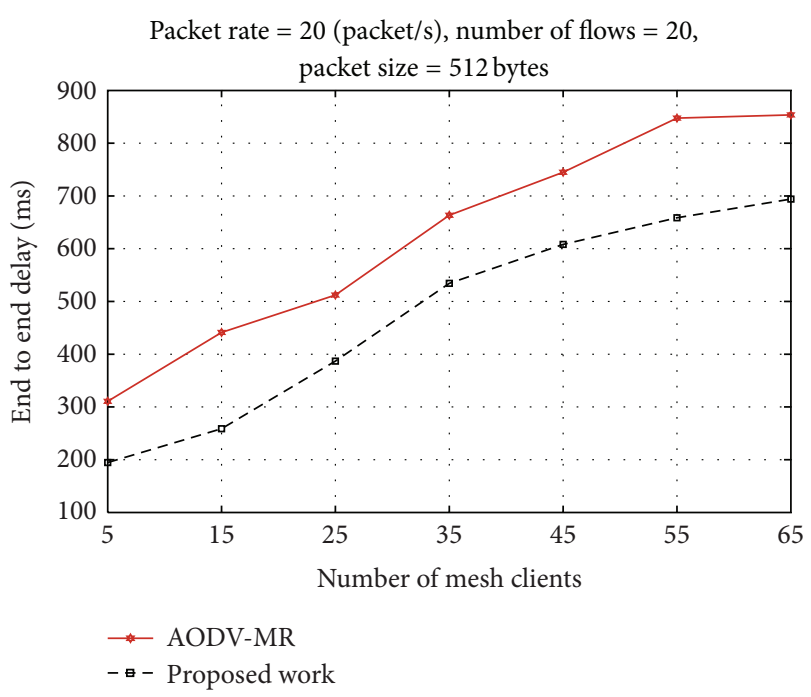

FIGURE 8: Number of mesh clients versus end to end delay.

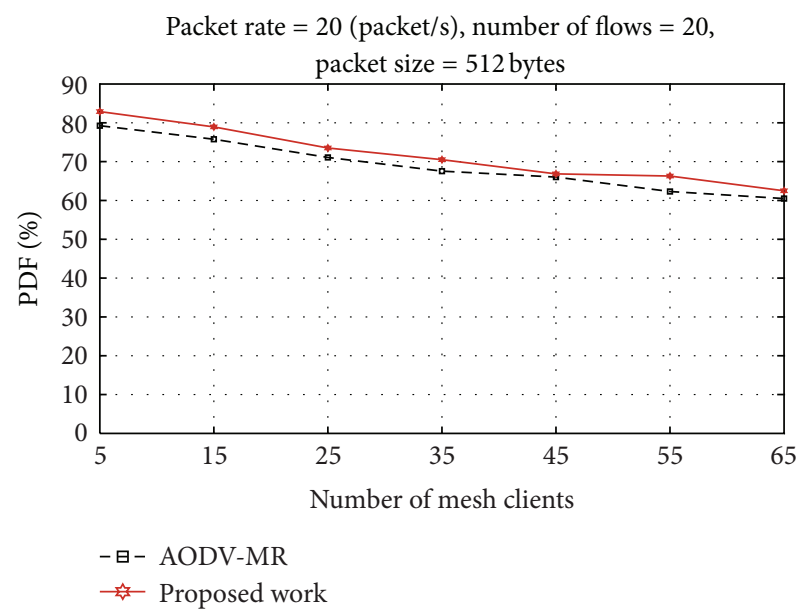

FIGURE 9: Number of mesh clients versus packet delivery fraction.

increase queue waiting time in each interface, consequently increasing end to end delay. Our proposed work selects less busy channel which helps to select path with less waiting time and less probability of packet collision. It uses single interface for sending control packet, and other interfaces can be utilized for sending data packet. As the number of packets in interface queue is decreased, our proposed work achieves significantly less delay compared with AODV-MR. For example when the number of mobile mesh clients is 65 , delays in our proposed work are $694.03 \mathrm{~ms}$ while in AODVMR are $853.66 \mathrm{~ms}$.

With the help of Figure 9, it is clear that as the number of mesh clients is increased packet delivery fraction (PDF) is decreased (i.e., inverse relationship). Because the number of lost packets increases due to highly collision at link layer and frequent movement of intermediate mesh clients, our proposed methodology shows an improvement over AODVMR. As the PDF in our proposed work is $66.28 \%$, AODV-MR is $62.31 \%$ when the number of mesh clients is 55 . 


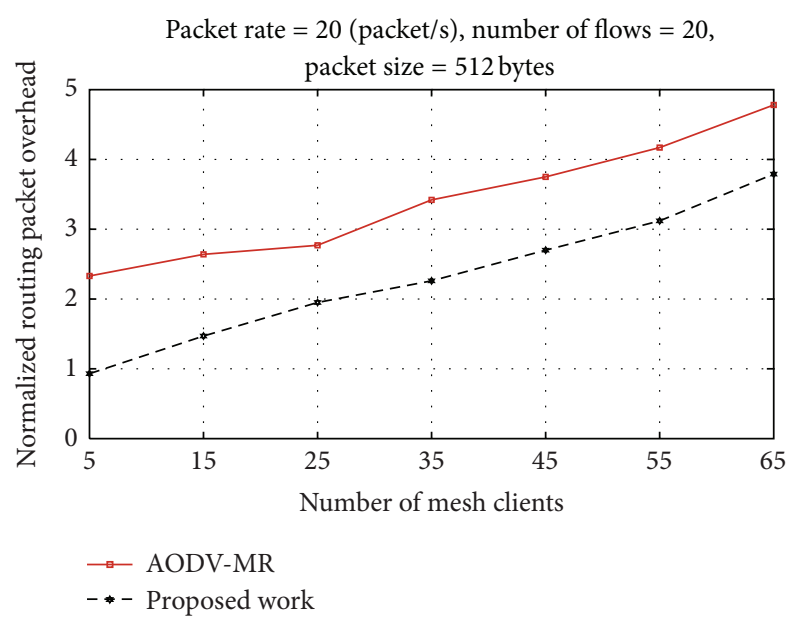

FIGURE 10: Number of mesh nodes versus normalized routing overhead.

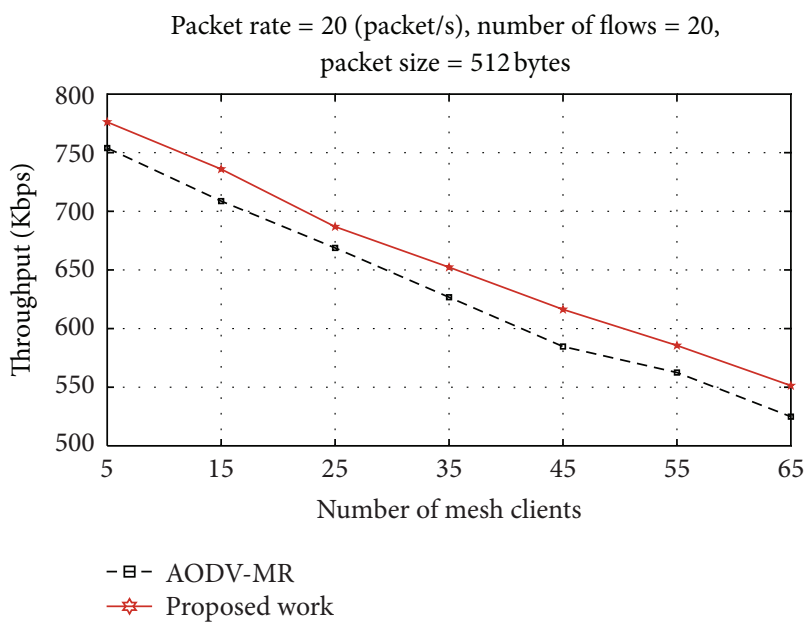

FIGURE 11: Throughput versus number of mesh clients.

Figure 10 shows that our proposed work is helpful to reduce the routing overhead packets when compared with AODV-MR. Since routing overhead is in direct relationship with the number of mesh clients, there is a link break, due to mobility of mesh clients and collision at link layer either between mesh routers or border mesh routers and mesh clients. This collision causes a link break, and subsequently error packets and route request packets will increase as the number of these links is broken. AODV-MR uses all interfaces to send these packets so that overhead will increase in overall network, while in the proposed work we are using single interface dynamically based on best channel, and hence overhead will reduced.

In Figure 11, the throughput has an inverse relationship with the number of nodes due to high loss of data packets and high consumes of bandwidth for sending routing packets again and again.

8.2. Scenario 2: Varying in Transmission Rate. Here, we vary transmission rate to study the impact of traffic load. The packet sending rate from 20 to 40 , while the number of flows

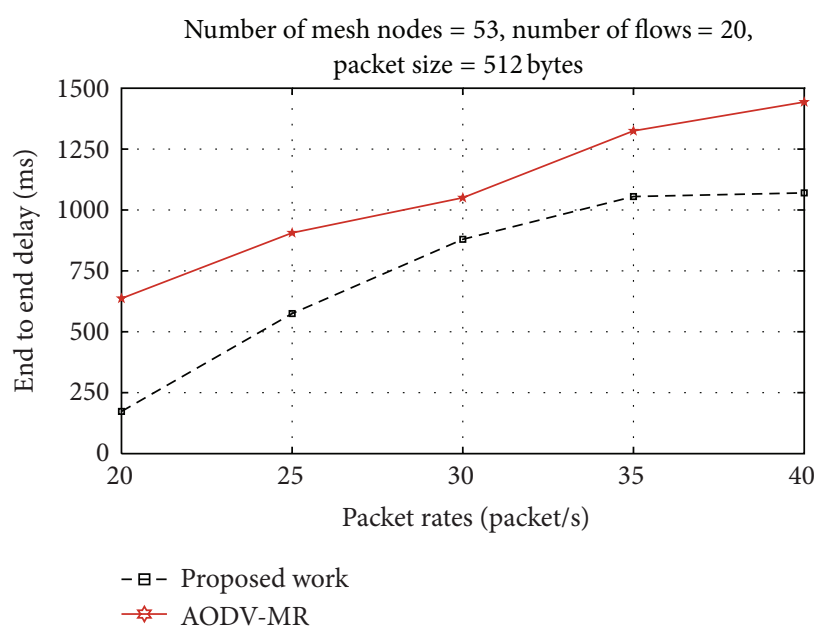

FIGURE 12: Packet rates versus end to end delay.

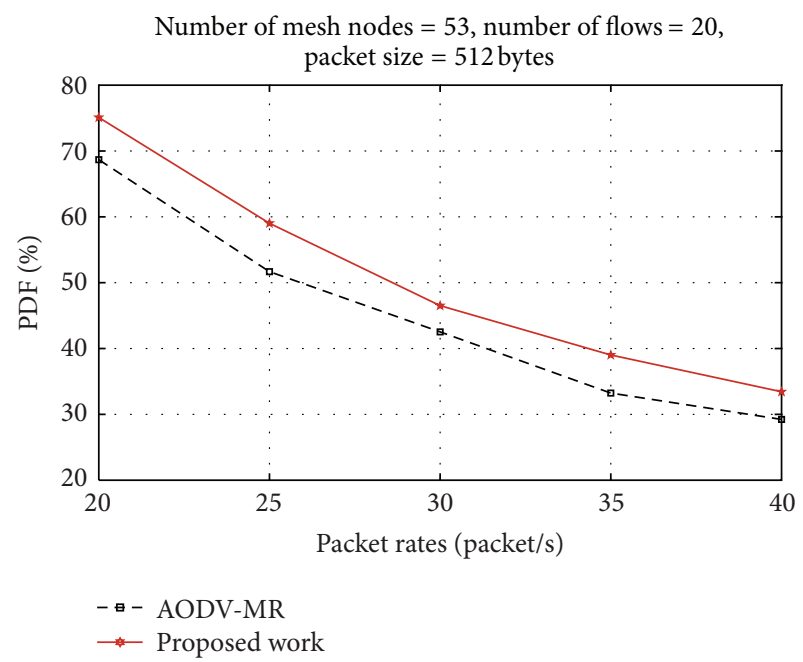

FIGURE 13: Packet rates versus packet delivery fraction.

from sources to destination is 20 and the number of mesh clients is 35 nodes (as shown in Figures 12, 13, 14, and 15).

In Figure 12, transmission rate has direct relationships with end to end delay. With the increment of traffic load the contention at link layer and back-off time at MAC layer will increase; this causes an increase in waiting time of packet in queue interface. It might cause an overflow queue problem and may prevent new arrival packets. As a result new packet will be lost, and retransmission mechanism will take place. This process will continue and may cause very high delay. Our proposed work has significantly achieved reduction of end to end delay over the AODV-MR. The AODV-MR uses all interfaces for sending multiple copies of control packets. The ADOV-MR does not have any criteria to choose an interface to send data packets to next hop, and the chosen interface may have full queue so that the packets will drop and retransmission will take place, while in our proposed work we are using single interface for sending control packets based on less channel utilization level. It will reduce waiting 


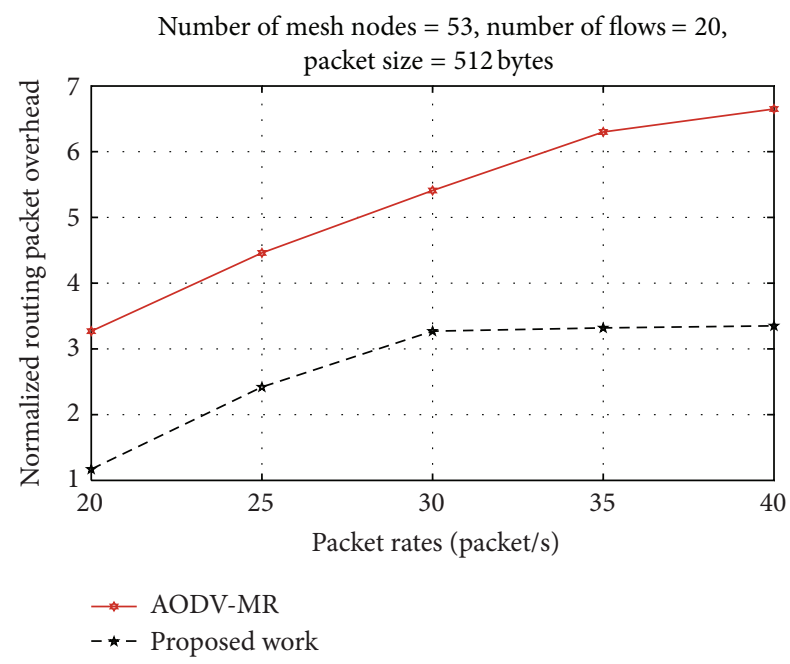

FIGURE 14: Normalized routing overhead versus packet rates (Packet/s).

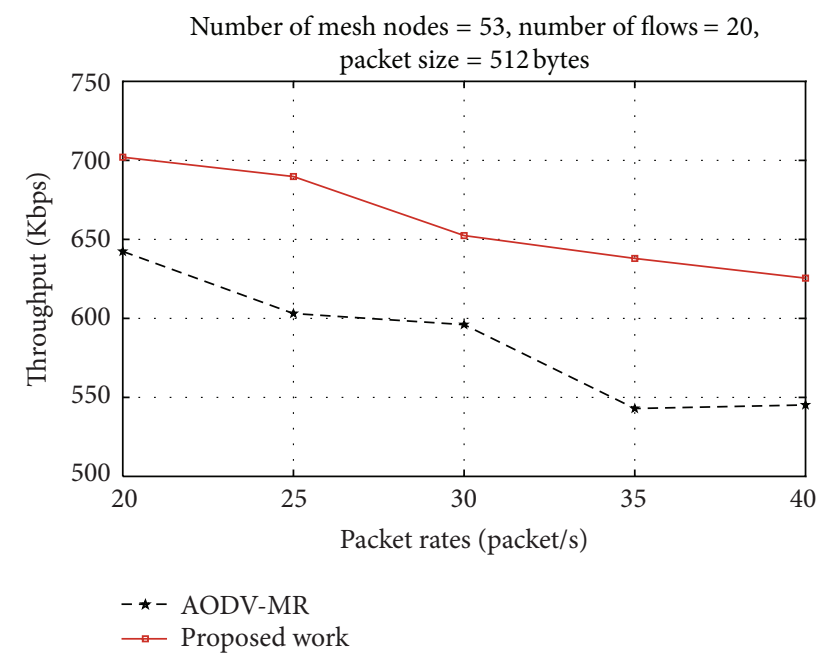

FIGURE 15: Throughput versus packet rates (packet/s).

time of packets in queue interface and thus eliminates the packet loss due to overflow problem. Consequently it reduces the number of retransmissions. For example in our proposed work the delay is $879.75 \mathrm{~ms}$ while in AODV-MR is $1050.1 \mathrm{~ms}$.

Figure 13 shows that our proposed work demonstrates a notable improvement in packet delivery fraction compared with AODV-MR in all packet rate ranges. Our methodology work manages to use multi-radio interfaces in proper manner.

With the help of Figure 14, we can observe that the routing overhead has direct relationship with packet rates. Our proposed work has a makeable reduction in routing overhead compared with AODV-MR. The main reason of that is that our proposed work uses single interface-based least channel utilization for sending packets. The AODV-MR broadcasts same copies of RREQ, same copies of hello messages, and same copies of RERR packets on all interfaces (i.e., channels)

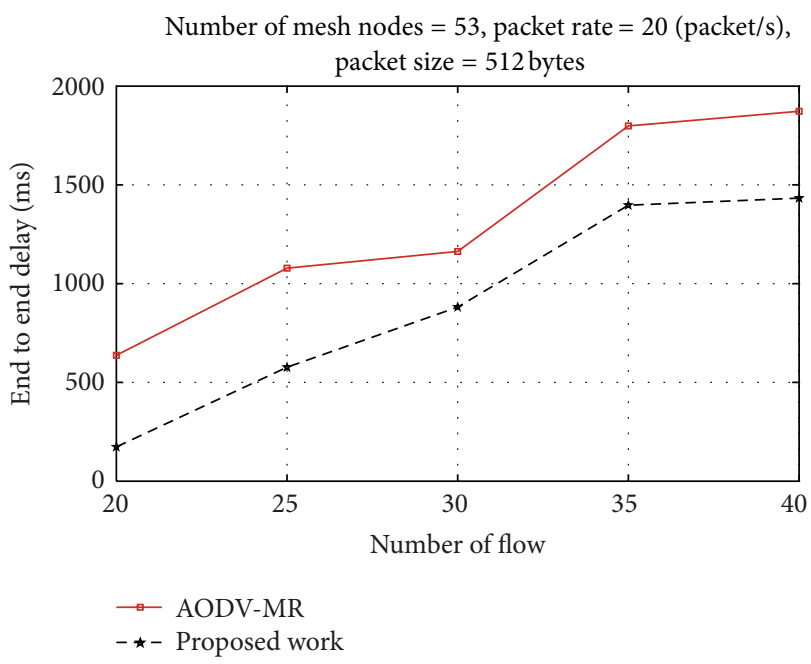

FIGURE 16: End to end delay versus number of flows.

at same time. We are using least channel contention to reduce the probability of packet collision.

As shown in Figure 15, the throughput has been affected by packet loss. These lost packets consume a bandwidth from hop to hop in path before it is dropped. However our proposed work has an apparent improvement over AODV-MR. It has been done by choosing lower contention in channel wireless medium.

8.3. Scenario 3: Varying Number of Flows. Here, we are evaluating the performance metric with the number of flows, varying from 20 to 40 in the number of flows. It is between mesh clients and gateways and among mesh clients themselves, the purpose of this study is to capacity-analyze the wireless network. The number of mesh nodes is fixed to 53 (18 mesh routers and 35 mesh clients) and the rate for transmission will be constant, 20 packets/s and it is shown in Figures 16, 17, 18, and 19.

As shown in Figure 16, end to end delay varies with the number of traffic flows. It is observed that, at lower traffic load, the end to end delay is low and it is increasing with traffic load. The contention time and retransmission time at MAC layer also increase. We have observed from Figure 16 that the proposed work has less end to end delay compared with AODV-MR. The AODV-MR does not use the channels in gateways and mesh routers efficiently, while in our proposed work we keep on continuously monitoring all channels, using less loaded channel to send packets.

As shown in Figure 17, we noted that packet delivery fraction is reaching a maximum value at lower traffic flow, decrementing with an increment in traffic flow, because channels reach saturation point. The AODV-MR does not have an efficient way to prevent packets from direct to highly contention channel either between mesh routers links or between mesh clients links in the path. It results in high loss packets and produces low packet delivery fraction, and our proposed work has better results than AODV-MR. Our proposed work route packets through less contention channel, so that 


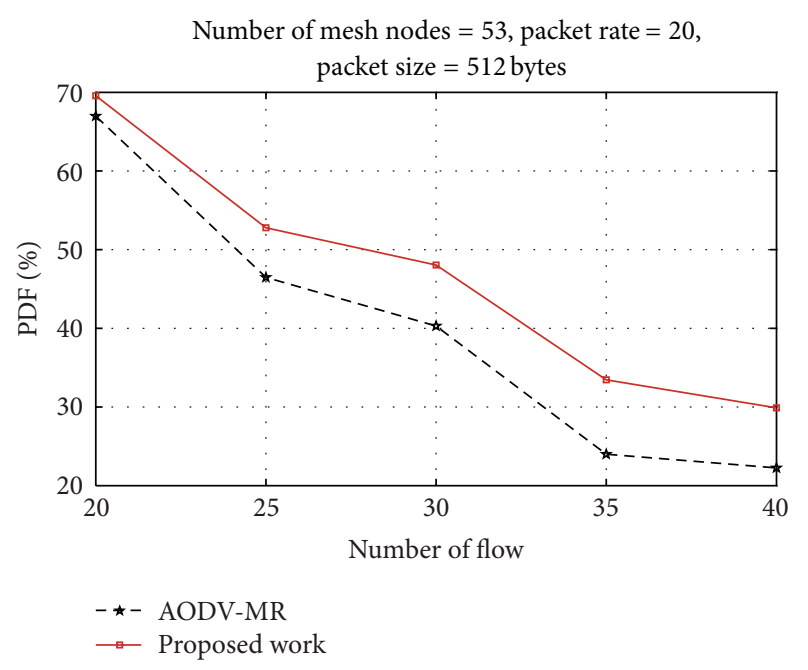

FIGURE 17: Packet delivery fraction versus number of flows.

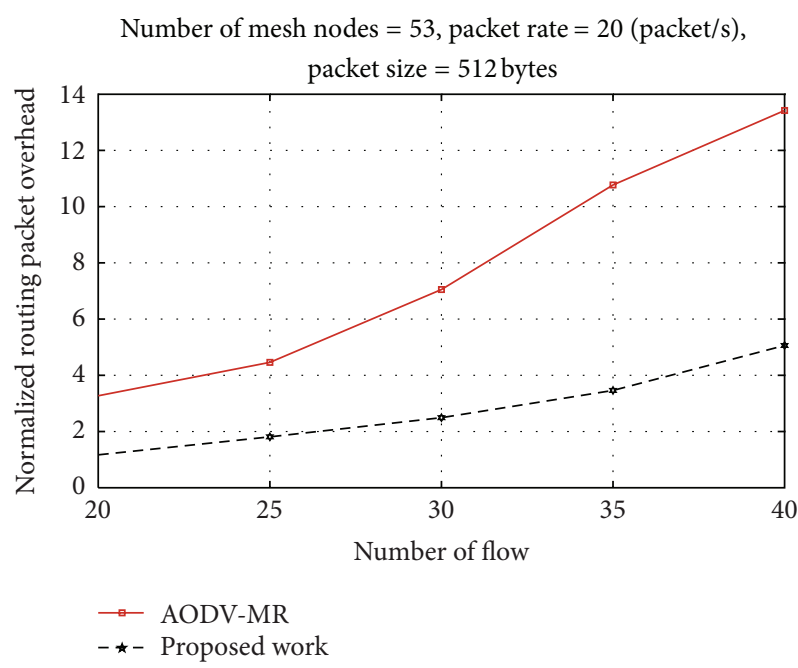

FIGURE 18: Number of flows versus normalized routing overhead.

the number of packets lost reduces packet delivery fraction increases when compared with AODV-MR.

Figure 18 shows that the route overhead increases when increasing flow. When the number of flows is 20 , the normalization routing overhead in AODV-MR is 3.8 while in our proposed work is about 1.8 .

Our proposed work achieves a lower routing overhead than AODV-MR. The AODV-MR protocol forms routes using random channels rather than selecting optimal channels available in the network. As a result, links frequently get saturated and suffer from interference, congestion resulting in routes break, and thus new route discoveries overhead is added. On the other hand, the proposed work selects a less contention channel (i.e., interfaces) to reduce a possibility of packet collisions; the waiting time of packets in the interface queue will reduce also.

Figure 19 shows that the throughput has an inverse relationship with the number of flows; it has been discussed previously.

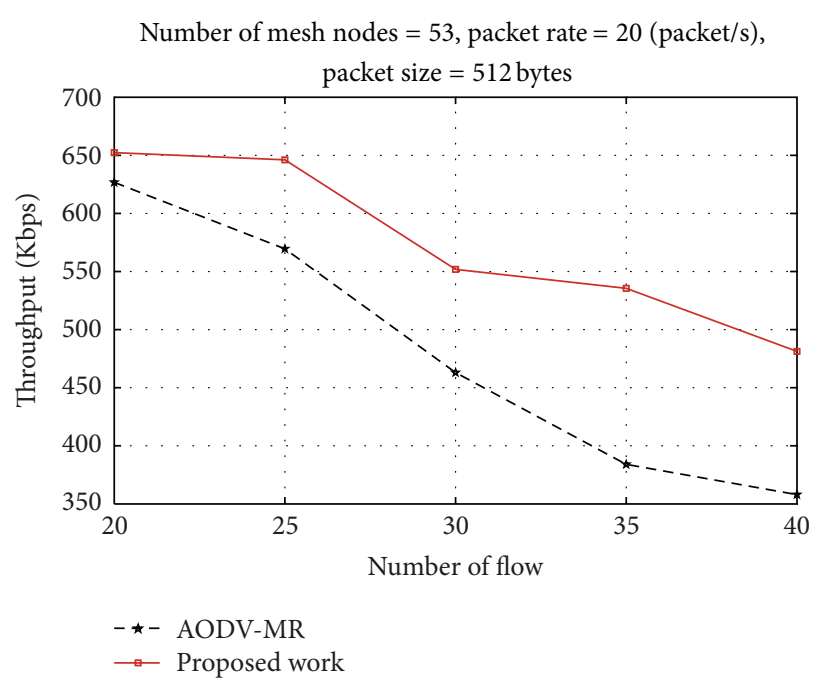

FIGURE 19: Throughput versus number of flow.

\section{Conclusion}

In this paper, we have proposed a mechanism for the enhancement of the hybrid wireless mesh network; the proposed work aims to reduce the overheads, helps select path with less probability of buffer overflow at queue interfaces, and helps to reduce it; it helps in improving load balance among multiple channels (interface) (i.e., less intraflow and interflow interference) between source and destination. The proposed work helps the gateway mesh routers and backbone mesh routers along a path to select the best interface (i.e., best channel) to send broadcasting packets (i.e., RREQ, RERR, and HELLO) and data packets. Our proposed work also provides a mechanism in a simple manner for assigning an interface during a traffic exchange between gateway and mesh client. Through our modification, we have reduced the number of broadcasting of control packet; data packets are being sent through the interface (i.e., channel) dynamically from hop to hop so that the possibility of using channel diverse is high. Though we have not addressed channel assignment problem, we have addressed routing overhead problem which is caused by using more than one interface to broadcast control packet. Our simple modifications result in a significant improvement in key performance metrics evaluated for hybrid WMNs.

\section{References}

[1] I. F. Akyildiz, X. Wang, and W. Wang, "Wireless mesh networks: a survey," Computer Networks, vol. 47, no. 4, pp. 445-487, 2005.

[2] A. A. Pirzada, M. Portmann, and J. Indulska, "Evaluation of multi-radio extensions to AODV for wireless mesh networks," in Proceedings of the 4th ACM International Workshop on Mobility Management and Wireless Access (MobiWAC '06), pp. 45-51, Torremolinos, Spain, October 2006.

[3] D. S. J. de Couto, High-throughput routing for multi-hop wireless networks [Ph.D. thesis], MIT, 2004.

[4] R. Draves, J. Padhye, and B. Zill, "Routing in multi-radio, multihop wireless mesh networks," in Proceedings of the 10th Annual 
International Conference on Mobile Computing and Networking (MobiCom '04), pp. 114-128, October 2004.

[5] I. F. Akyildiz and X. Wang, "Cross-layer design in wireless mesh networks," IEEE Transactions on Vehicular Technology, vol. 57, no. 2, pp. 1061-1076, 2008.

[6] C. E. Perkins and P. Bhagwat, "Highly dynamic destinationsequenced distance-vector routing (DSDV) for mobile computers," in Proceedings of the Conference on Communications Architectures, Protocols and Applications (SIGCOMM '94), pp. 234-244, October 1994.

[7] D. Johnson, D. Maltz, and Y. Hu, "The Dynamic Source Routing Protocol (DSR) for Mobile Ad Hoc Networks for IPv4," IETF, Request for Comment 4728, 2007.

[8] C. Perkins, E. Belding-Royer, and S. Das, "Ad hoc On-Demand Distance Vector (AODV) Routing," IETF, Request for Comment Request for Comments 3561, 2003.

[9] K. N. Ramach, R. M. M. Buddhikot, C. Girish, S. Miller, E. M. Belding-Royer, and K. C. Almeroth, "On the design and implementation of infrastructure mesh networks," in Proceedings of the IEEE Workshop on Wireless Mesh Networks, September 2005.

[10] P. Kyasanur and N. H. Vaidya, "Routing and link-layer protocols for multi-channel multi-interface ad hoc wireless networks," ACM SIGMOBILE Mobile Computing and Communications Review, vol. 10, no. 1, pp. 31-43, 2006.

[11] A. P. Subramanian, M. M. Buddhikot, and S. Miller, "Interference aware routing in multi-radio wireless mesh networks," in Proceedings of the 2nd IEEE Workshop on Wireless Mesh Networks (WiMESH '06), pp. 55-63, Reston, Va, USA, September 2006.

[12] T. Liu and W. Liao, "Capacity-aware routing in multi-channel multi-rate wireless mesh networks," in Proceedings of the IEEE International Conference on Communications (ICC '006), pp. 1971-1976, Istanbul, Turkey, July 2006.

[13] A. A. Pirzada, R. Wishart, and M. Portmann, "Multi-linked AODV routing protocol for wireless mesh networks," in Proceedings of the IEEE Global Telecommunications Conference (GLOBECOM '07), pp. 4925-4930, Washington, DC, USA, November 2007.

[14] A. A. Pirzada, R. Wishart, and M. Portmann, "ALARM: an adaptive load-aware routing metric for hybrid wireless mesh networks," in Proceedings of the 32nd Australasian Conference on Computer Science (ACSC '09), vol. 91, pp. 37-46.

[15] H. A. Mogaibel, M. Othman, S. Subramaniam, and N. A. W. Abdul Hamid, "On-demand channel reservation scheme for common traffic in wireless mesh networks," Journal of Network and Computer Applications, vol. 35, no. 4, pp. 1329-1351, 2012.

[16] C. E. Perkins, E. M. Royer, S. R. Das, and M. K. Marina, "Performance comparison of two on-demand routing protocols for Ad Hoc networks," IEEE Personal Communications, vol. 8, no. 1, pp. 16-28, 2001.

[17] IEEE Computer Society LAN/MAN Standards Committee, Wireless LAN Medium Access Control (MAC) and Physical Layer (PHY) Specifications: High-Speed Physical Layer in the $5 \mathrm{GHz}$ Band, IEEE Std. 802.11a-1999, The Institute of Electrical and Electronic Engineers, New York, NY, USA, 1999.

[18] IEEE Computer Society LAN/MAN Standards Committee, Wireless LAN Medium Access Control (MAC) and Physical Layer (PHY) Specifications: Higher-Speed Physical Layer Extension in the $2.4 \mathrm{GHz}$ Band, IEEE Std. 802.11b-1999, The Institute of Electrical and Electronic Engineers, New York, NY, USA, 1999.

[19] IEEE Computer Society LAN/MAN Standards Committee, Wireless LAN Medium Access Control (MAC) and Physical Layer
(PHY) Specifications: Amendment 4: Further Higher Data Rate Extension in the $2.4 \mathrm{GHz}$ Band, IEEE Std. 802.11g-2003, The Institute of Electrical and Electronic Engineers, New York, NY, USA, 2003.

[20] S. K. Biswash and C. Kumar, "Multi home agent and pointerbased (MHA-PB) location management scheme in integrated cellular-WLAN networks for frequent moving users," Computer Communications, vol. 33, no. 18, pp. 2260-2270, 2010.

[21] S. K. Biswash and C. Kumar, "An efficient metric-based (EMB) location management scheme for wireless cellular networks," Journal of Network and Computer Applications, vol. 34, no. 6, pp. 2011-2026, 2011.

[22] “The Network Simulator NS," http://www.isi.edu/nsnam/ns. 

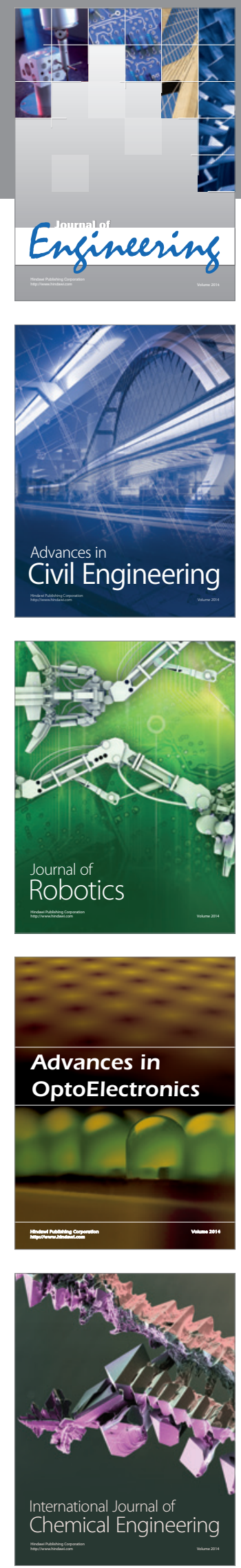

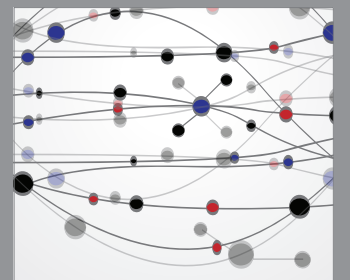

The Scientific World Journal
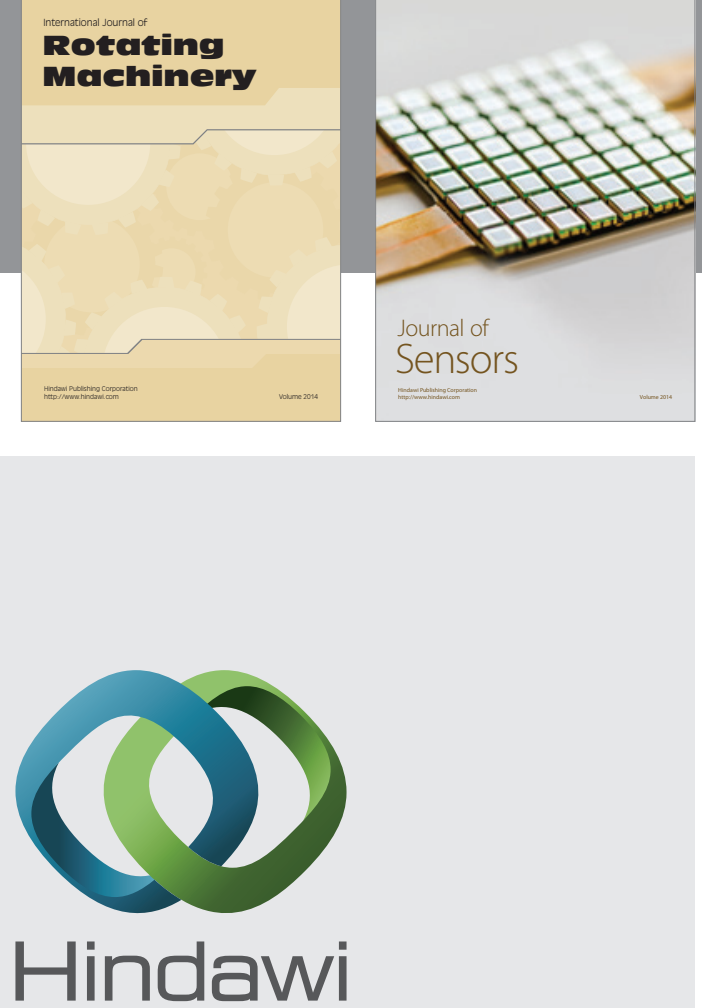

Submit your manuscripts at http://www.hindawi.com
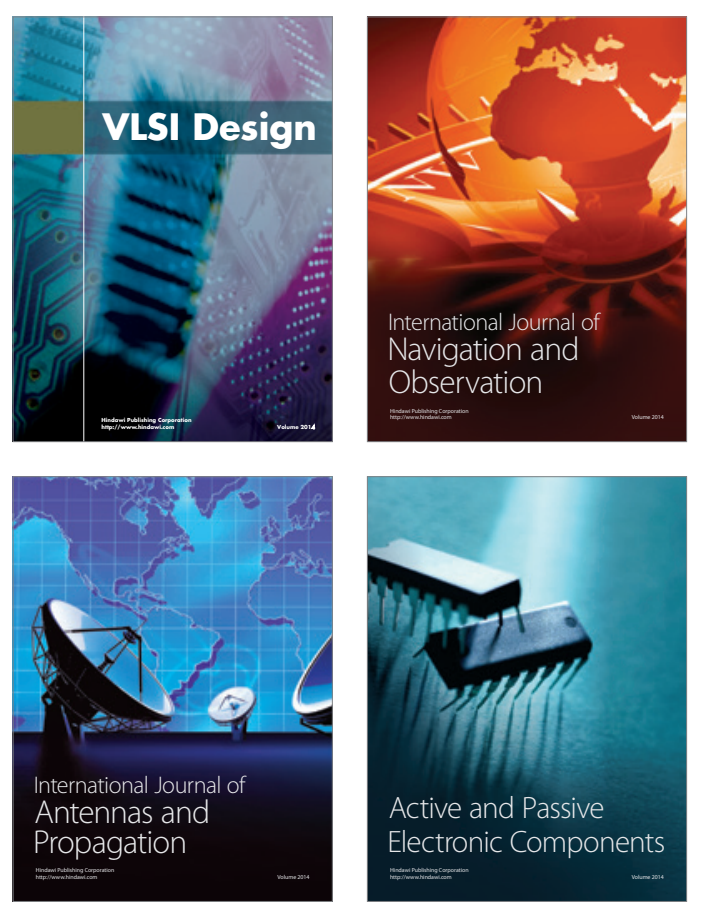
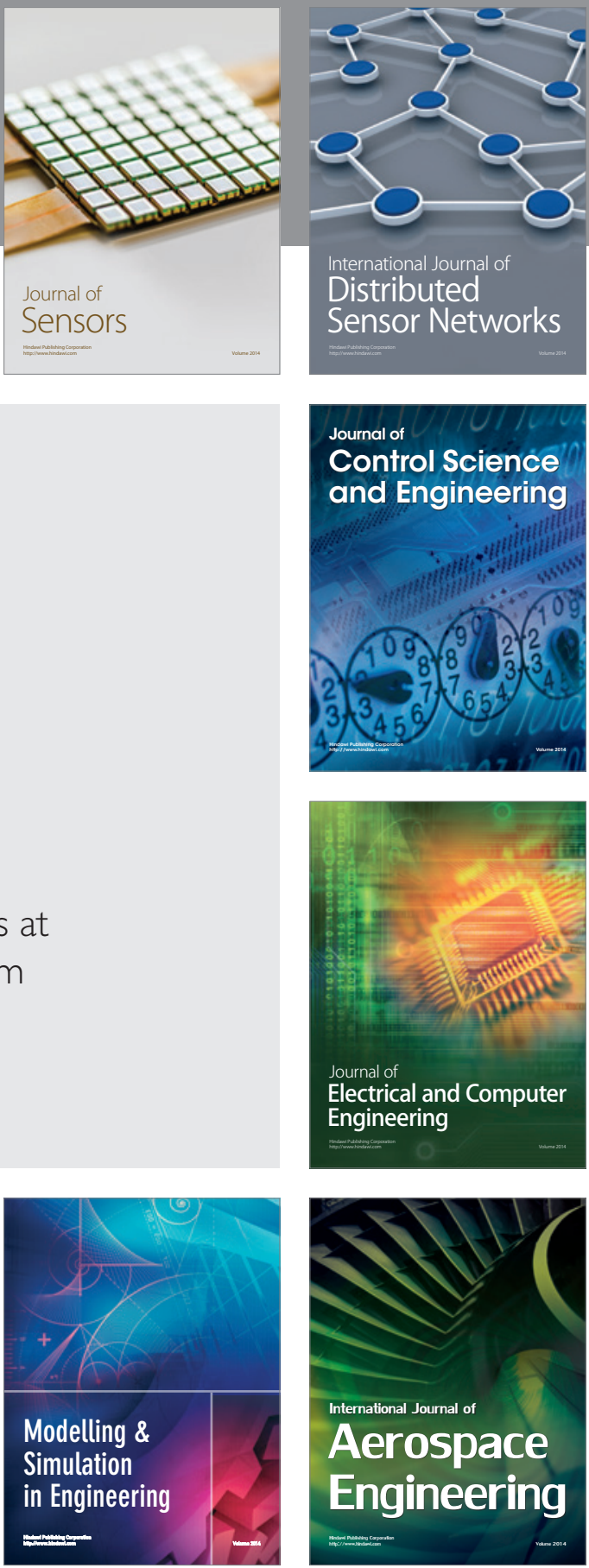

Journal of

Control Science

and Engineering
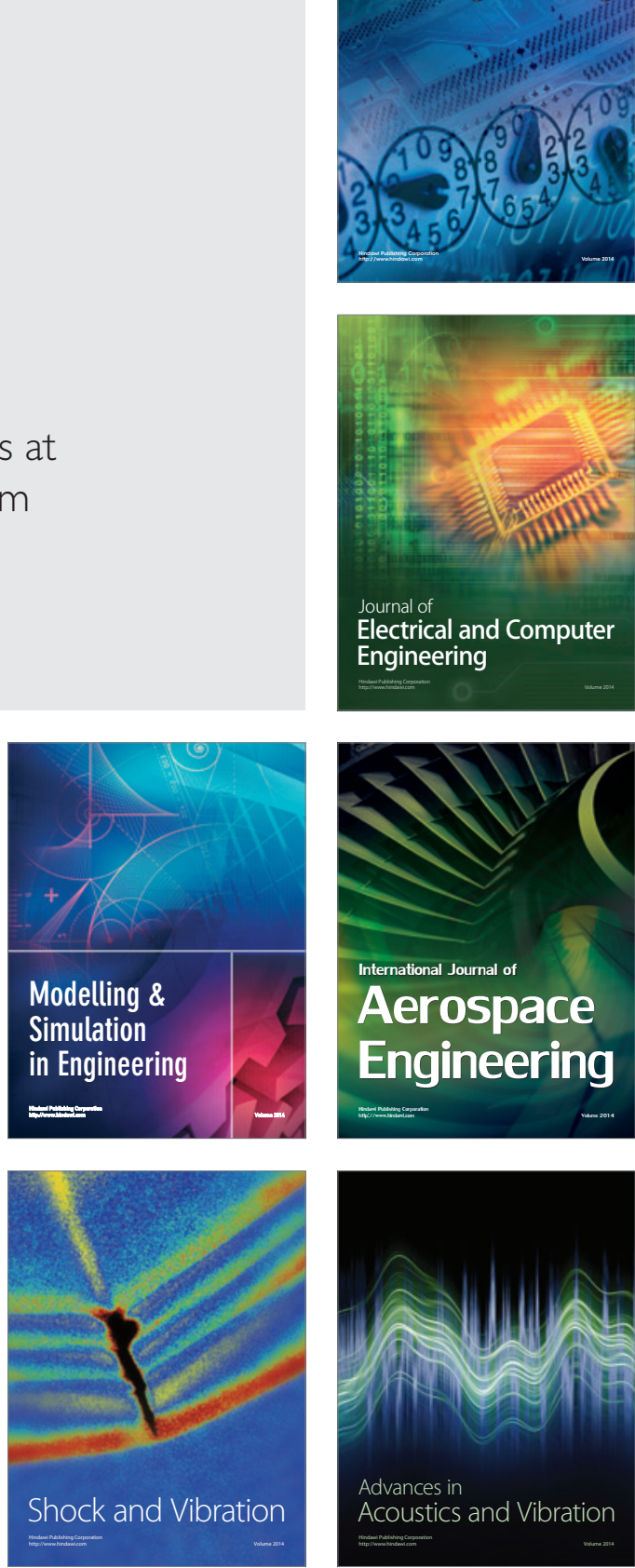